
\title{
continues to rise
}

The number of adults seeking orthodontic treatment in the UK

New figures released by The British Orthodontic Society (BOS), to coincide with National Smile Month, reveal the number of adults seeking orthodontic treatment in the UK continues to rise.

This survey, conducted in March 2018 among BOS members, was designed to gather new data about orthodontics and patient choices in the UK compared to two years ago.

Asked if they were seeing an increase in private adult treatment, $80 \%$ said yes. This figure compares to $75 \%$ in 2016 . When asked what kind of braces they provide to their patients, orthodontists revealed a cross section of approaches:

- Over 75\% supply fixed braces with clear aesthetic brackets

- Over 35\% supply lingual braces (fixed behind the teeth)

- Over $75 \%$ supply clear aligners.

The survey showed that adult patients are most likely to be female and in the 26 to 40 age bracket. However, the number of men seeking treatment appears to be on the rise. Nineteen percent of the respondents to the survey estimate that half of their adult patients are male. This compares to $13 \%$ in 2016 . The most popular system, provided by more than $98 \%$ of orthodontists, is fixed braces on the front of the teeth, often referred to as train tracks. This figure reflects the high number of young people treated as NHS patients for whom fixed braces is the most appropriate option. A quarter of BOS members responded to the survey. Of those who answered the survey, $27 \%$ see only NHS patients while $67 \%$ see both private and NHS patients.

\section{HMRC letters to associate dentists on employment status}

The BDA is aware that Her Majesty's Revenue and Customs (HMRC) has written recently to a number of associate dentists indicating that they are reviewing the employment status of associates.

The Association is advising dentists who receive a letter from HMRC to contact their financial adviser before responding.

The BDA is also requesting that dentists, including non-members, let them know if they have been contacted by HMRC. They should include a copy of the letter received if possible and an indication of their practising arrangements (eg NHS/private, and whether for example they work in a prototype practice).

You can scan or photograph the letter and email it to advice.enquiries@bda.org. Copies will be kept securely and destroyed after the work has concluded. The information provided will be used to help the Association build a picture and dentists' personal information will not be used.

The BDA is working with dental accountancy colleagues, will be monitoring developments closely and will offer further information and advice as and when there is more clarity around the scope of the HMRC process.

\section{The Assault}

H. S. Brand of the Academic Centre for Dentistry Amsterdam, and movie buff, continues his new series of columns on films featuring dentists with The Assault, a Dutch drama, directed by Fons Rademakers, and winner of the 1987 Academy Award for Best Foreign Language Film (1986).

The Dutch physician Anton Steenwijk is struggling through life with the question of why the Nazis killed his parents and brother. After he starts to suffer from severe toothache his wife Liesbeth calls their dentist Gerrit-Jan, an old student friend, who agrees to see Anton if he comes quickly. Liesbeth gives Anton a clove to help with the pain, 'Like they used to do in the East Indies'.

A little later Anton enters the dental practice and sits in the dental chair. The dentist says: 'Well, let's see friend, which one is it?' Anton points to a molar in the mandible, after which Gerrit-Jan pushes it with his finger. This causes Anton pain. Then Gerrit-Jan places occlusion paper in Anton's mouth and tells him to 'move back and forth gently'. Subsequently, Gerrit-Jan inspects the oral cavity with a dental mirror. 'I can see it already, he says.

When Gerrit-Jan picks a handpiece, Anton says, 'Professionally, I prefer an injection'. Gerrit-Jan indicates that there is absolutely no reason for that and reshapes the occlusal surface, using the hand piece. To Anton's surprise, the problems disappear immediately. Gerrit-Jan explains that the reason was an over-erupted molar. 'A matter of age. Now, just rinse and then we go'. Anton reacts in surprise: 'Go where?' It turns out that Gerrit-Jan had told Liesbeth that there was one condition for providing dental treatment on Saturday. In return Anton has to join him in an anti-nuclear protest, but Anton's wife did not tell him. Anton is furious, but still goes to the anti-nuclear demonstration. There Anton finally meets someone who can give him the answers to the question about the tragic night during World War II. 